Research article

Open Access

\title{
Synthesis of New $\boldsymbol{N}, \boldsymbol{N}$-Bis(5-arylidene-4-oxo-
} 4,5-dihydrothiazolin-2-yl)piperazine Derivatives

Under Mic rowave Irradiation and Preliminary Biological Evaluation

\author{
Wacothon Karime Coulibaly ${ }^{1,2}$, Ludovic PaQuin ${ }^{1}$, Anoubilé BÉniÉ ${ }^{2}$, \\ Yves-Alain BEKRO ${ }^{2}$, Emilie DURIEUX ${ }^{3}$, Laurent MEIJER ${ }^{4}$, \\ Rémy Le GuÉVel ${ }^{5}$, Anne Corlu ${ }^{5}$, Jean-Pierre BAZUREAU * ${ }^{1}$
}

\footnotetext{
${ }^{1}$ Université de Rennes 1, Institut des Sciences Chimiques de Rennes (ISCR), UMR CNRS 6226, groupe Ingénièrie Chimique et Molécules pour le Vivant (ICMV), Bât. 10A, campus de Beaulieu, Avenue du Général Leclerc, CS 74205, 35042 Rennes Cedex, France.

${ }^{2}$ Université d'Abobo-Adjamé, Laboratoire de Chimie Bioorganique et de Subtances Naturelles (LCBSN), BP 802, Abidjan 02, République de la Côte d'Ivoire.

${ }^{3}$ Protein Phosphorylation and Human Disease Group, Station Biologique CNRS, Place G. Tessier, BP 74, 29682 Roscoff, France.

${ }^{4}$ ManRos Therapeutics (From Sea to Pharmacy), Hôtel de Recherche, Centre de Perharidy, 29680 Roscoff, France.

${ }^{5}$ Université de Rennes 1, ImPACcell Platform, IFR 140, Bât. 8, 2 Avenue du Prof. Léon Bernard, CS 34317, 35043 Rennes Cedex, France.
}

* Corresponding author. E-mail: jean-pierre.bazureau@univ-rennes1.fr (J.-P. Bazureau)

Sci Pharm. 2012; 80: 825-836

doi:10.3797/scipharm.1206-04

Published: $\quad$ September $16^{\text {th }} 2012$

Accepted: $\quad$ September $16^{\text {th }} 2012$

Received: June $4^{\text {th }} 2012$

This article is available from: http://dx.doi.org/10.3797/scipharm.1206-04

(c) Coulibaly et al.; licensee Österreichische Apotheker-Verlagsgesellschaft m. b. H., Vienna, Austria.

This is an Open Access article distributed under the terms of the Creative Commons Attribution License (http://creativecommons.org/licenses/by/3.0/), which permits unrestricted use, distribution, and reproduction in any medium, provided the original work is properly cited.

\begin{abstract}
New $N, N^{\prime}$-bis(5-arylidene-4-oxo-4,5-dihydrothiazoline-2-yl)diamine derivatives $\mathbf{5}$ were prepared in two steps from rhodanine and piperazine, or 1,4-bis(3-aminopropyl)piperazine, under microwave reaction conditions with retention of configuration. Some of these compounds were tested for in vitro antiproliferative activities and for their kinase inhibitory potencies towards six kinases (CDK5/p25, GSK3a/ß, DYRK1A, DYRK2, CLK1, and CLK2). The compound 5d showed nanomolar activity towards DYRK1A kinase $\left(\mathrm{IC}_{50}=0.041 \mu \mathrm{M}\right)$.
\end{abstract}

\section{Keywords}

5-Arylidene rhodanine • 2,2'-(Piperazine-1,4-diyl)bis(5-benzylidene-1,3-thiazol-4(5H)-one) • Microwave irradiation $\bullet$ Cytotoxity $\bullet$ Protein kinase $\bullet$ Diamines 


\section{Introduction}

The 2-amino-5-arylidene-5H-thiazol-4-ones and their 5-arylidene rhodanine precursors are a class of five-membered heterocyclic rings (FMHRs) considered as "privileged scaffolds" in the medicinal chemistry community [1]. Considerable work has been published over decades about their chemistry and biology. A number of compounds containing the 2-amino-5-arylidenethiazol-4(5H)-one moiety have been shown to exhibit antiinflammatory [2], antimicrobial [3], and antitumor [4] effects. Among these compounds, Darbufelone ${ }^{\circledR}$ A [5] (Figure 1 ) is orally active in animal models of inflammation [6] and DBPT $\mathbf{B}$ is under clinical trials for colon cancer [7]. 5-Arylidene rhodanines have also proven to be attractive for the discovery of new candidates. A series of rhodanine-based hits $\mathbf{C}$ (Figure 1) were found as potent and selective inhibitors of the "atypical" dualspecificity phosphatase (DSP) family member-JNK-stimulating phophatase-1 (JSP-1). Compounds of this class may be useful for the treatment of inflammatory and proliferative disorders [8]. As the last example, epalrestat D reduced the symptoms of diabetic neuropathy [9].<smiles>CC(C)(C)c1cc(/C=C2\SC(N)=NC2=O)cc(C(C)(C)C)c1O</smiles>

A<smiles>O=C(O)c1cccc(N2C(=O)/C(=C/c3ccc(F)cc3)SC2=S)c1</smiles>

C<smiles>O=C1N=C(Nc2ccccc2)S/C1=C\c1cc(O)cc(O)c1</smiles>

B<smiles>CC(=C\c1ccccc1)/C=C1/SC(=S)N(CCC(=O)O)C1=O</smiles>

D

Fig. 1. Structures of Darbufelone (A), DBPT (B), inhibitor of DSP (C), and epalrestat (D).

Protein kinases are the enzymes which control the phosphorylation of protein in cellular life [10] which is frequently deregulated in human diseases. For the pharmaceutical industry, the protein kinases represent interesting targets for new therapeutic agents [11] and this interest was boosted by the approval of the first marketed inhibitor Gleevec ${ }^{\mathrm{TM}}$ used in myeloid leukemia [12]. Due to the biological activity associated with the 2-aminothiazolidinone moiety, we decided in this paper to explore the synthesis of $N, N$ '-bis(5arylidene-4-oxo-4,5-dihydrothiazolidin-2-yl)diamines derived from piperazine or 1,4-bis(3aminopropyl)piperazine as linkers, and to study their effects on cells and protein kinases. 


\section{Results and Discussion}

\section{Chemistry}

The strategy used for the synthesis of the symmetric derivatives $\mathbf{5}$ is outlined in Scheme 1. The reactions have been realized under microwave irradiation [13]. The main benefits of microwave irradiation technology are the significant rate-enhancements and sometimes elevated product yields enabling the rapid synthesis of molecules of potential value in medicinal chemistry [14]. The synthesis started by the solution-phase Knoevenagel condensation of aryl aldehydes 2 and commercial rhodanine 1a. The expected compounds 3a-c were prepared in yields ranging from 65 to $88 \%$ with a reaction time of 10 min. under microwave irradiation (MWI) at $65^{\circ} \mathrm{C}$ in the presence of sodium acetate. In a similar approach, the 5 -arylidene rhodanine propanoic derivatives $\mathbf{3} \mathbf{d}-\mathbf{g}$ were easily synthesized using solvent-less reaction conditions under microwave irradiation $\left(130^{\circ} \mathrm{C}, 10 \mathrm{~min}\right.$.). The geometric double bond of $\mathbf{3}$ was attributed as being $Z$ by the shielding effect of the carbonyl group $\mathrm{C}-4$ on the olefinic proton $\mathrm{H}-5(\delta 7.5 \mathrm{ppm})$ in the ${ }^{1} \mathrm{H}$ NMR spectra.<smiles>[R7]N1C(=O)CSC1=S</smiles><smiles>[R]c1ccc(C=O)cc1[R]</smiles>

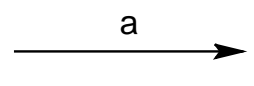<smiles>[R]c1ccc(/C=C2\SC(=S)N([R])C2=O)cc1[R]</smiles>

3a $\mathrm{R}^{1}=\mathrm{R}^{2}=\mathrm{R}^{3}=\mathrm{H}$

3b $R^{1}=R^{2}=H, R^{3}=M e O$

3c $\mathrm{R}^{1}=\mathrm{H}, \mathrm{R}^{2}, \mathrm{R}^{3}=\mathrm{OCH}_{2} \mathrm{O}$

3d $\mathrm{R}^{1}=\left(\mathrm{CH}_{2}\right)_{2} \mathrm{CO}_{2} \mathrm{H}, \mathrm{R}^{2}, \mathrm{R}^{3}=\mathrm{H}$

3e $\mathrm{R}^{1}=\left(\mathrm{CH}_{2}\right)_{2} \mathrm{CO}_{2} \mathrm{H}, \mathrm{R}^{2}=\mathrm{H}, \mathrm{R}^{3}=\mathrm{MeO}$

3f $\mathrm{R}^{1}=\left(\mathrm{CH}_{2}\right)_{2} \mathrm{CO}_{2} \mathrm{H}, \mathrm{R}^{2}, \mathrm{R}^{3}=\mathrm{OCH}_{2} \mathrm{O}$

3g $\mathrm{R}^{1}=\left(\mathrm{CH}_{2}\right)_{2} \mathrm{CO}_{2} \mathrm{H}, \mathrm{R}^{2}, \mathrm{R}^{3}=\mathrm{O}\left(\mathrm{CH}_{2}\right)_{2} \mathrm{O}$

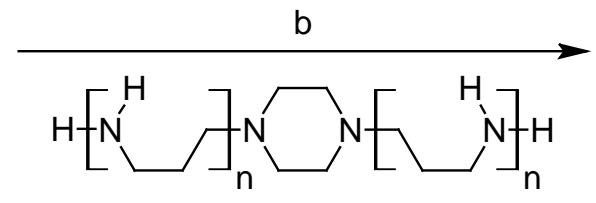

4a $n=0 ; 4 b \quad n=1$

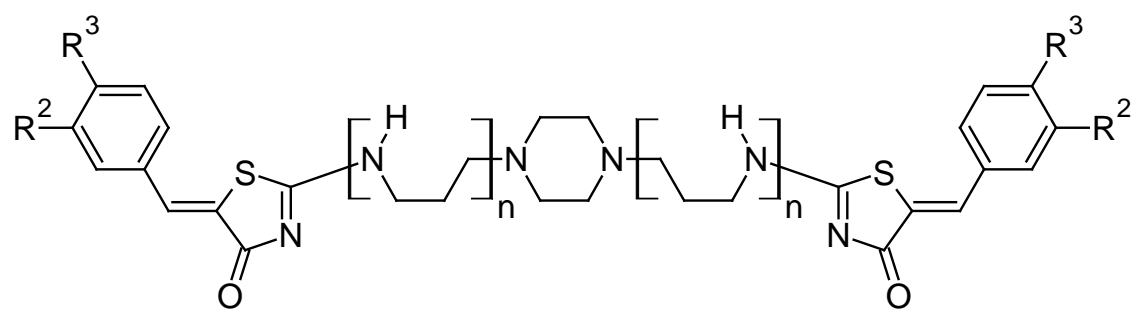

5a $R^{2}=R^{3}=H, n=0$

5b $R^{2}=H, R^{3}=M e O, n=0$

5c $\mathrm{R}^{2}, \mathrm{R}^{3}=\mathrm{OCH}_{2} \mathrm{O}, \mathrm{n}=0$

5d $R^{2}=\mathrm{H}, \mathrm{R}^{3}=\mathrm{MeO}, \mathrm{n}=1$

5e $\mathrm{R}^{2}, \mathrm{R}^{3}=\mathrm{OCH}_{2} \mathrm{O}, \mathrm{n}=1$

Sch. 1. a) for 3a-c: $\mathrm{MeOH}, \mathrm{AcONa} 3$ eq., $\mathrm{MWI}, 65^{\circ} \mathrm{C}, 10 \mathrm{~min}$. and for $\mathbf{3 d - g}$ : $\mathrm{MWI}$, $130^{\circ} \mathrm{C}, 10 \mathrm{~min}$. b) $\mathrm{MWI}, 80-120^{\circ} \mathrm{C}, 30 \mathrm{~min}$. 
Transformation of 5-arylidene rhodanine 3 into 2-amino-5-arylidene-5 $\mathrm{H}$-thiazol-4-one after addition of a primary amine [15] usually involves activation of the $\mathrm{C}=\mathrm{S}$ bond of rhodanine via the thioether intermediate that subsequently undergoes a thioalkyl/nitrogen displacement. In order to be able to carry out such sulfur/nitrogen displacement in a faster and more efficient way - avoiding the preparation of the thioether intermediate - we examined the influence of microwave irradiation on the reaction between compound $\mathbf{3}$ and the symmetric diamino linkers $\mathbf{4 a}, \mathbf{b}$. The experiments revealed that optimal reaction conditions were obtained at $80-120^{\circ} \mathrm{C}$ after 30 minutes. It is noteworthy that sulfur/nitrogen displacement reactions at $120^{\circ} \mathrm{C}$ under microwave irradiation have been realized in solution with hexane to avoid decomposition of compound $\mathbf{5}$. The desired compounds 5a-e were prepared in poor to moderate yields (13-26\%) and their structures were substantiated by ${ }^{1} \mathrm{H},{ }^{13} \mathrm{C} N M R$, and HRMS analyses and only the more thermodynamically stable $Z$-isomers were obtained.

\section{Biology}

To evaluate the potency of the compounds $\mathbf{3 a - c}, \mathbf{5 a}$, and $\mathbf{5 c}$ for their in vitro antiproliferative activities, we used six representative tumor cell lines of liver (Huh7), colon (Caco2, HCT 116), breast (MDA-MB 231), prostate (PC3), lung ( $\mathrm{NCl}$ ), and one normal cell line (fibroblats) and measured survival. Results are reported in Table 1 . The 5-arylidene rhodanines $\mathbf{3 a - c}$ and two compounds $\mathbf{5 a}$ and $\mathbf{5 c}$ showed measurable, albeit very poor, cytotoxic activity. No clear tendency for higher activity of these compounds can be deduced from these results. Since the $\log P$ values of all tested compounds are in the same range, influences of lipophilicity on cytotoxic activity cannot be deduced as well.

Tab. 1. Cell effects of the products and calculated partition coefficients log $P$ (calculated with Chem Draw Pro, Cambridge Soft)

\begin{tabular}{|c|c|c|c|c|c|c|c|c|}
\hline \multirow[b]{2}{*}{ Cpd. } & \multicolumn{8}{|c|}{ Cell lines $I_{50}(\mu M)$} \\
\hline & Huh7 & Caco2 & $\begin{array}{c}\text { MDA-MB } \\
231\end{array}$ & $\begin{array}{l}\mathrm{HCT} \\
116\end{array}$ & PC3 & $\mathrm{NCl}$ & Fibroblats & $\log P_{\text {calc. }}$ \\
\hline $3 \mathbf{a}$ & $>25$ & $>25$ & $>25$ & $>25$ & $>25$ & $>25$ & $>25$ & 2.0 \\
\hline $3 b$ & $>25$ & $>25$ & $>25$ & $>25$ & $>25$ & $>25$ & $>25$ & 1.9 \\
\hline $3 c$ & $>25$ & $>25$ & $>25$ & $>25$ & $>25$ & $>25$ & $>25$ & 1.8 \\
\hline $5 \mathbf{a}$ & $>25$ & 25 & $>25$ & $>25$ & $>25$ & $>25$ & $>25$ & 4.8 \\
\hline $5 c$ & $>25$ & 20 & $>25$ & $>25$ & 40 & $>25$ & 40 & 5.3 \\
\hline Roscovitine & 10 & 10 & 15 & 8 & 8 & 20 & $>25$ & - \\
\hline DMSO & $>25$ & $>25$ & $>25$ & $>25$ & $>25$ & $>25$ & $>25$ & - \\
\hline
\end{tabular}

The kinase inhibitory potencies of compounds $\mathbf{3 a - c}$ and $\mathbf{5 d}$ were evaluated as $\mathrm{IC}_{50}$ values towards six protein kinases (CDK5/p25, GSK3a/ $\beta$, DYRK1A, DYRK2, CLK1 and CLK2) and the results are reported in Table 2 . The 5 -arylidene rhodanines $\mathbf{3} \mathbf{a}, \mathbf{b}$ appeared to be inactive towards these six protein kinases, but interesting results were obtained with compounds 5d and 3c. The 1,4-bis[(5Z)-5-(4-methoxybenzylidene)methylene-4-oxo-4,5dihydrothiazol-2-yl]piperazine $\mathbf{5 b}$ and the 5-arylidene rhodanine $3 \mathbf{c}$ are very active on DYRK1A [16] and a noteworthy $\mathrm{IC}_{50}=41 \mathrm{nM}$ was measured for $\mathbf{5 b}$. The compound $\mathbf{5 d}$ has also shown submicromolar inhibition potencies towards DYRK2 $\left(\mathrm{IC}_{50}=0.6 \mu \mathrm{M}\right)$ and CLK1 $\left(\mathrm{IC}_{50}=0.5 \mu \mathrm{M}\right)$. Regarding these results, the potential of the $(5 Z) 5$-arylidene-4-0xo-4,5- 
dihydro-thiazolidine-2-yl moiety appended on 1,4-bis(3-aminopropyl)piperazine $\mathbf{4 b}$ as a linker could be highly interesting in the development of a new class of inhibitors of DYRK1A kinase which is known to be involved in Alzheimer's disease/Down syndrome [17].

Tab. 2. CDK5/p25, GSK3a/ $\quad$, DYRK1A, DYRK2, CLK1, CLK3 inhibitions.

\begin{tabular}{lcccccc}
\hline \multirow{2}{*}{ Cpd. } & \multicolumn{7}{c}{ IC $_{50}(\boldsymbol{\mu M M})$} \\
\cline { 2 - 7 } & CDK5/p25 & GSK3 $\boldsymbol{\alpha} / \boldsymbol{\beta}$ & DYRK1A & DYRK2 & CLK1 & CLK2 \\
\hline $\mathbf{3 a}$ & $>10$ & $>10$ & $>10$ & $>10$ & $>10$ & $>10$ \\
3b & $>10$ & $>10$ & $>10$ & $>10$ & $>10$ & $>10$ \\
3c & $>10$ & 8.5 & 0.070 & - & - & - \\
5d & $>10$ & $>10$ & 0.041 & 0.6 & 0.5 & 7.7 \\
\hline
\end{tabular}

\section{Conclusion}

In conclusion, we worked out a short and practical synthesis under microwave irradiation of $\quad N, N^{\prime}$-(5-arylidene-4-oxo-4,5-dihydrothiazolidin-2-yl)diamines $5 a-e$ derived from piperazine $\mathbf{4 a}[18,19]$ and 1,4-bis(3-aminopropyl)piperazine $\mathbf{4 b}$. The in vitro antiproliferative activities are extremely weak and this could be due to a lower cellular penetration of compounds $\mathbf{3}$ and $\mathbf{5}$ or a lower interaction with the cellular targets. Surprisingly, the compound $\mathbf{5} \mathbf{d}$ has shown nanomolar inhibition potency towards DYRK1A and this interesting inhibition led us to expand our efforts in the synthesis of diversely disubstituted $N, N^{\prime}$-bispiperazine derivatives with the 5-arylidene thiazolidinone moiety as new potential inhibitors of this kinase. Work is in progress to gain deeper insight into the structure-activity relationships (SAR) of this new interesting class of diamines.

\section{Experimental}

\section{General}

Elemental analysis: Flash EA1112 CHN/O Thermo Electron; HRMS (MS/MS ZABSpec Tof Micromass, EBE TOF geometry, IP $8 \mathrm{eV})$; NMR: BRUKER AC $300 \mathrm{P}\left({ }^{1} \mathrm{H}: 300 \mathrm{MHz},{ }^{13} \mathrm{C}: 75\right.$ $\mathrm{MHz}$; melting points: Leica System Kofler VMHB Melting Point apparatus (not corrected); microwave reactor: Monowave 300 Anton-Paar $(850 \mathrm{~W})$, monowave software package.

\section{General procedure I: Preparation of 5-arylidene-2-thioxo-thiazolidine-4-one 3a-c under microwave irradiation.}

A mixture of rhodanine $1 \mathrm{a}(1 \mathrm{~g}, 7.5 \mathrm{mmol})$, aldehyde $2(8.95-9 \mathrm{mmol})$, sodium acetate $(1.85 \mathrm{~g}, 22.55 \mathrm{mmol})$, and methanol $(5 \mathrm{ml})$ was placed in a borosilicate glass vial $(10 \mathrm{ml})$ with a Teflon ${ }^{\circledR}$ magnetic stir bar and sealed with a snap cap. The glass tube was then introduced into the Monowave 300 Anton-Paar microwave cavity ( $P=850$ Watt) and the stirred mixture was irradiated at $65^{\circ} \mathrm{C}$ (with a power of 10 Watt) for 30 min. After microwave dielectric heating, the crude reaction mixture was allowed to cool down to room temperature, and then was filtered on a Buchner funnel and the insoluble compound $\mathbf{3}$ was washed with methanol $(2 \times 5 \mathrm{ml})$. The crude compound 3 was purified by recrystallization 
from methanol and further dried under high vacuum ( $10^{-2}$ Torr) for 1 hour, which gave the desired compound $\mathbf{3}$ as a powder.

\section{General procedure II: Preparation of 3-(5-arylidene-4-oxo-2-thioxo-thiazolidine-3- yl)propanoic acid $3 d-g$ under solvent-free microwave irradiation.}

A mixture of rhodanine $\mathbf{1 b}(0.5 \mathrm{~g}, 2.44 \mathrm{mmol})$ and aldehyde $2(2.44 \mathrm{mmol})$ was placed in a borosilicate glass vial $(10 \mathrm{ml})$ with a Teflon ${ }^{\circledR}$ magnetic stir bar and sealed with a snap cap. The glass tube was then introduced into the Monowave 300 Anton-Paar microwave cavity $(P=850$ Watt $)$ and the stirred mixture was irradiated at $130^{\circ} \mathrm{C}$ for $10 \mathrm{~min}$. After microwave dielectric heating, the crude reaction mixture was allowed to cool down to room temperature and $5 \mathrm{ml}$ of a mixture of ethanol/hexane (1:1) were added directly to the glass vial. The resulting precipitated product 3 was filtered on a Buchner funnel and the insoluble compound 3 was washed with the same mixture $(2 \times 5 \mathrm{ml})$. The crude compound $\mathbf{3}$ was further dried under high vacuum $\left(10^{-2}\right.$ Torr) for 1 hour, which gave the desired compound 3 as a powder.

\section{General procedure III: Synthesis of N,N'-bis(5-arylidene-4-oxo-4,5-dihydro- thiazolidine-2-yl)piperazine derivatives 5a-e under microwave irradiation.}

A mixture of 5-arylidene-2-thioxo-thiazolidine-4-one 3 (4 mmol), piperazine $4 \mathbf{a}$ (172 $\mathrm{mg}, 2$ mmol) or 1,4-bis(3-aminopropyl)piperazine 4 b $(400 \mathrm{mg}, 2 \mathrm{mmol}$ ) was placed in a borosilicate glass vial $(10 \mathrm{ml})$ with a Teflon ${ }^{\circledR}$ magnetic stir bar and sealed with a snap cap. The glass tube was then introduced into the Monowave 300 Anton-Paar microwave cavity $\left(P=850\right.$ Watt) and the stirred mixture was irradiated at $80-120^{\circ} \mathrm{C}$ (with a power of $5-200$ Watt) for $30 \mathrm{~min}$. After microwave dielectric heating, the crude reaction mixture was allowed to cool down to room temperature and $10 \mathrm{ml}$ of cooled ethanol $\left(4^{\circ} \mathrm{C}\right)$ were added directly in the glass vial. The resulting precipitated product 5 was filtered off, washed with 2 $x 5 \mathrm{ml}$ of ethanol and dried under high vacuum $\left(10^{-2}\right.$ Torr) at room temperature for 1 hour. After ${ }^{1} \mathrm{H}$ NMR analysis, the product 5 was purified by recrystallization from methanol, which gave the desired compound $\mathbf{3}$ as a powder.

\section{(5Z)-5-Benzylidene-2-thioxo-1,3-thiazolidin-4-one (3a)}

Prepared following General procedure I from benzaldehyde $\mathbf{2 a}(0.95 \mathrm{~g}, 8.95 \mathrm{mmol})$ to give $1.08 \mathrm{~g}(65 \%)$ as an orange powder. $\mathrm{Mp}=208-210^{\circ} \mathrm{C} .{ }^{1} \mathrm{H}$ NMR $\left(300 \mathrm{MHz}, \mathrm{DMSO}-d_{6}\right) \delta$ $(\mathrm{ppm})=7.44-7.55(\mathrm{~m}, 5 \mathrm{H}, \mathrm{Ar}), 7.57(\mathrm{~s}, 1 \mathrm{H},=\mathrm{CH}), 13.12(\mathrm{br} \mathrm{s}, 1 \mathrm{H}, \mathrm{NH}) .{ }^{13} \mathrm{C}$ NMR $(75 \mathrm{MHz}$, DMSO- $\left.d_{6}\right) \delta(\mathrm{ppm})=126.88(\mathrm{C}-1), 129.20(\mathrm{C}-2), 130.30(\mathrm{C}-3), 130.37(\mathrm{C}-4), 130.45(\mathrm{C}=$, C-5), $133.22(=\mathrm{CH}), 171.28$ (C=S, C-2); 196,64 (C=O, C-4). HRMS, $m / z=243.9868$ found (calculated for $\mathrm{C}_{10} \mathrm{H}_{7} \mathrm{NONaS}_{2},[\mathrm{M}+\mathrm{Na}]^{+}$requires 243.9867). Anal. Calcd. for $\mathrm{C}_{10} \mathrm{H}_{7} \mathrm{NOS}_{2}$ : C, 54.27; H, 3.19. Found: C, 54.23; H, 3.16.

\section{(5Z)-5-(4-Methoxybenzylidene)-2-thioxo-1,3-thiazolidin-4-one (3b)}

Prepared following General procedure I from 4-methoxybenzaldehyde $\mathbf{2 b}(1.23 \mathrm{~g}, 9 \mathrm{mmol})$ to give $1.36 \mathrm{~g} \mathrm{(74 \% )}$ as an orange powder. $\mathrm{Mp}=250-252^{\circ} \mathrm{C} .{ }^{1} \mathrm{H}$ NMR $(300 \mathrm{MHz}, \mathrm{DMSO}-$ $\left.d_{6}\right) \delta(\mathrm{ppm})=3.82\left(\mathrm{~s}, 3 \mathrm{H}, \mathrm{OCH}_{3}\right), 7.10(\mathrm{~d}, \mathrm{~J}=8.8 \mathrm{~Hz}, 2 \mathrm{H}, \mathrm{H}-3, \mathrm{Ar}), 7.55(\mathrm{~d}, J=9.1 \mathrm{~Hz}, 2 \mathrm{H}$, $\mathrm{H}-2, \mathrm{Ar}), 7.57(\mathrm{~s}, 1 \mathrm{H} ;=\mathrm{CH}), 13.76(\mathrm{br} \mathrm{s}, 1 \mathrm{H}, \mathrm{NH}) \cdot{ }^{13} \mathrm{C} \mathrm{NMR}\left(75 \mathrm{MHz}, \mathrm{DMSO}-d_{6}\right) \delta(\mathrm{ppm})=$ $55.45\left(\mathrm{OCH}_{3}\right), 114.92\left(\mathrm{C}-3^{\prime}\right), 124.90(\mathrm{C}=, \mathrm{C}-5), 126.02\left(\mathrm{C}-1^{\prime}\right), 129.71(=\mathrm{CH}), 132.28\left(\mathrm{C}-2{ }^{\prime}\right)$, 160.82 (C-4'), 173.024 (C=S, C-2), 197.25 (C=O, C-4). HRMS, $m / z=295.9792$ found 
(calculated for $\mathrm{C}_{11} \mathrm{H}_{9} \mathrm{NO}_{2} \mathrm{Na}_{2} \mathrm{~S}_{2}, \quad[\mathrm{M}-\mathrm{H}+2 \mathrm{Na}]^{+}$requires 295.9792). Anal. Calcd. for $\mathrm{C}_{11} \mathrm{H}_{9} \mathrm{NO}_{2} \mathrm{~S}_{2}$ : C, 52.57; $\mathrm{H}, 3.61$. Found: $\mathrm{C}, 52.65 ; \mathrm{H}, 3.67$.

\section{(5Z)-5-(1,3-Benzodioxol-5-ylmethylidene)-2-thioxo-1,3-thiazolidin-4-one (3c)}

Prepared following General procedure I from piperonaldehyde $2 \mathrm{c}(1.35 \mathrm{~g}, 9 \mathrm{mmol})$ to give $1.74 \mathrm{~g}(88 \%)$ as an orange powder. $\mathrm{Mp}>260^{\circ} \mathrm{C} .{ }^{1} \mathrm{H}$ NMR $\left(300 \mathrm{MHz}, \mathrm{DMSO}-d_{6}\right) \delta(\mathrm{ppm})=$ $6.13\left(\mathrm{~s}, 2 \mathrm{H} ; \mathrm{OCH}_{2} \mathrm{O}\right), 7.11-7.45(\mathrm{~m}, 3 \mathrm{H} ; \mathrm{H}-5$ ', H-6', Ar), $7.52(\mathrm{~s}, 1 \mathrm{H},=\mathrm{CH}), 7.55(\mathrm{~s}, 1 \mathrm{H}, \mathrm{H}-$ 2'; Ar), 13.74 (br s, $1 \mathrm{H}, \mathrm{NH}) .{ }^{13} \mathrm{C}$ NMR $\left(75 \mathrm{MHz}, \mathrm{DMSO}-d_{6}\right) \delta(\mathrm{ppm})=102.10\left(\mathrm{OCH}_{2} \mathrm{O}\right)$, 109.26 (C=, C-5), 109.45 (C-6'), 123.02 (C-1'), 126.65 (C-5'), 127.16 (=CH), 131.81 (C-2'), 148.27 (C-4'), 149.60 (C-3'), 169.58 (C=S, C-2), 195.51 (C=O, C-4). HRMS, m/z = 287.9765 found (calculated for $\mathrm{C}_{11} \mathrm{H}_{7} \mathrm{NO}_{3} \mathrm{NaS}_{2},[\mathrm{M}+\mathrm{Na}]^{+}$requires 287.9765). Anal. Calcd. for $\mathrm{C}_{11} \mathrm{H}_{7} \mathrm{NO}_{3} \mathrm{~S}_{2}$ : C, 49.80; $\mathrm{H}, 2$.66. Found: $\mathrm{C}, 49.91 ; \mathrm{H}, 2.69$.

\section{3-[(5Z)-5-Benzylidene-4-oxo-2-thioxo-1,3-thiazolidin-3-yl]propanoic acid (3d)}

Prepared following General procedure II from benzaldehyde $2 \mathbf{a}(0.256 \mathrm{~g}, 2.44 \mathrm{mmol})$ to give $3 \mathbf{d}$ in $60 \%$ yield as a brown powder. $\mathrm{Mp}=224-226^{\circ} \mathrm{C} .{ }^{1} \mathrm{H}$ NMR $\left(300 \mathrm{MHz}, \mathrm{DMSO}-d_{6}\right)$ $\delta(\mathrm{ppm})=2.33\left(\mathrm{~m}, 2 \mathrm{H}, \mathrm{CH}_{2}\right), 4.15\left(\mathrm{~m}, 2 \mathrm{H}, \mathrm{CH}_{2}\right), 7.51-7.65(\mathrm{~m}, 5 \mathrm{H}, \mathrm{H}-2, \mathrm{H}-3, \mathrm{H}-4, \mathrm{Ar})$, $7.80(\mathrm{~s}, 1 \mathrm{H},=\mathrm{CH}), 13.16(\mathrm{br} \mathrm{s}, 1 \mathrm{H}, \mathrm{OH}) .{ }^{13} \mathrm{C} \mathrm{NMR}\left(75 \mathrm{MHz}, \mathrm{DMSO}-d_{6}\right) \delta(\mathrm{ppm})=33.30$ $\left(\mathrm{CH}_{2}\right), 41.78\left(\mathrm{CH}_{2}\right), 122.6(\mathrm{C}=, \mathrm{C}-5), 129.46\left(\mathrm{C}-2{ }^{\prime}\right), 130.57$ (C-3'), 130.84 (C-4'), 132.63 $(=\mathrm{CH}), 133.02(\mathrm{C}-1), 166.76(\mathrm{C}=\mathrm{O}, \mathrm{C}-4), 171.94\left(\underline{\mathrm{CO}}{ }_{2} \mathrm{H}\right), 193.15(\mathrm{C}=\mathrm{S}, \mathrm{C}-2) . \mathrm{HRMS}, \mathrm{m} / \mathrm{z}=$ 337.9904 found (calculated for $\mathrm{C}_{13} \mathrm{H}_{10} \mathrm{NO}_{3} \mathrm{Na}_{2} \mathrm{~S}_{2},[\mathrm{M}-\mathrm{H}+2 \mathrm{Na}]^{+}$requires 337.9898).

\section{3-[(5Z)-5-(4-Methoxybenzylidene)-4-oxo-2-thioxo-1,3-thiazolidin-3-yl]propanoic acid} (3e)

Prepared following General procedure II from 4-methoxybenzaldehyde 2b (0.332 g, 2.44 $\mathrm{mmol})$ to give $3 \mathrm{e}$ in $60 \%$ as a brown powder. $\mathrm{Mp}>260^{\circ} \mathrm{C}$. ${ }^{1} \mathrm{H}$ NMR $\left(300 \mathrm{MHz}, \mathrm{DMSO}-d_{6}\right) \delta$ $(\mathrm{ppm})=2.24\left(\mathrm{t}, 2 \mathrm{H}, \mathrm{J}=8.2 \mathrm{~Hz}, \mathrm{CH}_{2}\right), 3.83\left(\mathrm{~s}, 3 \mathrm{H}, \mathrm{OCH}_{3}\right), 4.12\left(\mathrm{t}, 2 \mathrm{H}, \mathrm{J}=8.2 \mathrm{~Hz}, \mathrm{CH}_{2}\right)$, $7.10(\mathrm{~d}, 2 \mathrm{H}, \mathrm{J}=8.8 \mathrm{~Hz}, \mathrm{H}-3, \operatorname{Ar}), 7.64(\mathrm{~d}, 2 \mathrm{H}, \mathrm{J}=8.8 \mathrm{~Hz}, \mathrm{H}-2, \operatorname{Ar}), 7.86(\mathrm{~s}, 1 \mathrm{H},=\mathrm{CH})$, 13.26 (br s, $1 \mathrm{H}, \mathrm{OH}) .{ }^{13} \mathrm{C}$ NMR $\left(75 \mathrm{MHz}, \mathrm{DMSO}-d_{6}\right) \delta(\mathrm{ppm})=34.11\left(\mathrm{CH}_{2}\right), 42.20\left(\mathrm{CH}_{2}\right)$, $55.56\left(\mathrm{OCH}_{3}\right), 115.11$ (C-3'), 119.26 (C=, C-5), 125.58 (C-1'); $129.90(=\mathrm{CH}), 132.84\left(\mathrm{C}-2^{\prime}\right)$, 161.41 (C-4'), $166.86(\mathrm{C}=\mathrm{O}, \mathrm{C}-4), 171.98\left(\mathrm{CO}_{2} \mathrm{H}\right), 192.86(\mathrm{C}=\mathrm{S}, \mathrm{C}-2) . \mathrm{HRMS}, \mathrm{m} / \mathrm{z}=$ 346.0185 found (calculated for $\mathrm{C}_{14} \mathrm{H}_{13} \mathrm{NO}_{4} \mathrm{NaS}_{2}$, [M+Na] ${ }^{+}$requires 346.0184).

\section{3-[(5Z)-5-(1,3-Benzodioxol-5-ylmethylidene)-4-oxo-2-thioxo-1,3-thiazolidin- 3-yl]propanoic acid (3f)}

Prepared following General procedure II from piperonal $\mathbf{2 c}(0.366 \mathrm{~g}, 2.44 \mathrm{mmol})$ to give $\mathbf{3 f}$ in $63 \%$ as a brown powder. $\mathrm{Mp}=220-224^{\circ} \mathrm{C} .{ }^{1} \mathrm{H}$ NMR $\left(300 \mathrm{MHz}, \mathrm{DMSO}-d_{6}\right) \delta(\mathrm{ppm})=$ $2.61\left(\mathrm{t}, 2 \mathrm{H}, \mathrm{J}=3.9 \mathrm{~Hz}, \mathrm{CH}_{2}\right), 4.21\left(\mathrm{t}, 2 \mathrm{H}, \mathrm{J}=4.7 \mathrm{~Hz}, \mathrm{CH}_{2}\right), 6.15\left(\mathrm{~s}, 2 \mathrm{H}, \mathrm{OCH}_{2} \mathrm{O}\right), 7.09-$ $7.23(\mathrm{~m}, 3 \mathrm{H}, \mathrm{H}-2, \mathrm{H}-5, \mathrm{H}-6, \mathrm{Ar}), 7.74(\mathrm{~s}, 1 \mathrm{H},=\mathrm{CH}), 12.50$ (br s, $1 \mathrm{H}, \mathrm{OH}) .{ }^{13} \mathrm{C}$ NMR $(75$ $\left.\mathrm{MHz}, \mathrm{DMSO}-d_{6}\right) \delta(\mathrm{ppm})=30.74\left(\mathrm{CH}_{2}\right), 40.30\left(\mathrm{CH}_{2}\right), 102.21\left(\mathrm{OCH}_{2} \mathrm{O}\right), 109.35\left(\mathrm{C}-2^{\prime}\right)$, 109.61 (C-5'), 119.71 (C=, C-5), 126.98 (C-6'), 127.14 (C-1'), $133.21(=\mathrm{CH}), 148.35$ (C-4'), $149.88\left(\mathrm{C}-3^{\prime}\right), 166.67(\mathrm{C}=\mathrm{O}, \mathrm{C}-4), 171.72\left(\mathrm{CO}_{2} \mathrm{H}\right), 192.97(\mathrm{C}=\mathrm{S}, \mathrm{C}-2) . \mathrm{HRMS}, \mathrm{m} / \mathrm{z}=$ 346.0185 found (calculated for $\mathrm{C}_{14} \mathrm{H}_{13} \mathrm{NO}_{4} \mathrm{NaS}_{2}$, [M+Na] ${ }^{+}$requires 346.0184).

\section{3-[(5Z)-5-(2,3-Dihydro-1,4-benzodioxin-6-ylmethylidene)-4-oxo-2-thioxo- 1,3-thiazolidin-3-yl]propanoic acid (3g)}

Prepared following General procedure II from 1,4-benzodioxane-6-carboxaldehyde (2d) 
(0.401 $\mathrm{g}, 2.44 \mathrm{mmol}$ ) to give $\mathbf{3 g}$ in $54 \%$ yield as a brown powder. $\mathrm{Mp}=226-228^{\circ} \mathrm{C} .{ }^{1} \mathrm{H}$ NMR (300 MHz, DMSO- $\left.d_{6}\right) \delta(\mathrm{ppm})=2.55\left(\mathrm{t}, 2 \mathrm{H}, \mathrm{J}=7.3 \mathrm{~Hz}, \mathrm{CH}_{2}\right), 4.18(\mathrm{t}, 2 \mathrm{H}, \mathrm{J}=7.4 \mathrm{~Hz}$, $\left.\mathrm{CH}_{2}\right), 4.30\left(\mathrm{~m}, 4 \mathrm{H}, \mathrm{OCH}_{2} \mathrm{CH}_{2} \mathrm{O}\right), 7.02(\mathrm{~d}, 1 \mathrm{H}, \mathrm{J}=8.6 \mathrm{~Hz}, \mathrm{H}-5, \mathrm{Ar}), 7.12(\mathrm{~d}, 1 \mathrm{H}, \mathrm{J}=2.2 \mathrm{~Hz}$, $\mathrm{H}-6, \mathrm{Ar}), 7.15(\mathrm{~s}, 1 \mathrm{H}, \mathrm{H}-2, \mathrm{Ar}), 7.70(\mathrm{~s}, 1 \mathrm{H} ;=\mathrm{CH}), 12.46(\mathrm{br} \mathrm{s}, 1 \mathrm{H}, \mathrm{OH}) .{ }^{13} \mathrm{C} \mathrm{NMR}(75 \mathrm{MHz}$, DMSO-d $\left.d_{6}\right) \delta(\mathrm{ppm})=31.30\left(\mathrm{CH}_{2}\right), 40.30\left(\mathrm{CH}_{2}\right), 64.01\left(\mathrm{OCH}_{2} \mathrm{CH}_{2} \mathrm{O}\right), 118.22\left(\mathrm{C}-2^{\prime}\right), 119.47$ (C-5'), 119.79 (C=, C-5), 124.46 (C-6'), 126.31 (C-1'), 132.96 (=CH), 143.82 (C-4'), 146.25 (C-3'); 166.67 (C=O, C-4), $171.80\left(\mathrm{CO}_{2} \mathrm{H}\right), 192.93(\mathrm{C}=\mathrm{S}, \mathrm{C}-2)$. HRMS, $\mathrm{m} / \mathrm{z}=374.0138$ found (calculated for $\mathrm{C}_{15} \mathrm{H}_{13} \mathrm{NO}_{5} \mathrm{NaS}_{2},[\mathrm{M}+\mathrm{Na}]^{+}$requires 374.0133).

(5Z,5'Z)-2,2'-(Piperazine-1,4-diyl)bis(5-benzylidene-1,3-thiazol-4(5H)-one) (1,4-Bis[(5Z)-5-benzylidene-4-oxo-4,5-dihydro-1,3-thiazol-2-yl]piperazine, 5a)

Prepared following General procedure III from 5-benzylidene-2-thioxothiazolidin-4-one (3a) (885 mg, $4 \mathrm{mmol})$ and piperazine $4 \mathrm{a}(172 \mathrm{mg}, 2 \mathrm{mmol})$ in hexane $(2 \mathrm{ml})$ at $120^{\circ} \mathrm{C}$ to give $5 \mathbf{a}$ in $13 \%$ yield as a brown powder. $\mathrm{Mp}>260^{\circ} \mathrm{C}$. ${ }^{1} \mathrm{H}$ NMR $(300 \mathrm{MHz}$, DMSO-d $) \delta(\mathrm{ppm})=$ 3.34 (br s, 8H, N(CH $\left.{ }_{2}{ }_{4} \mathrm{~N}\right), 7.28-7.7\left(\mathrm{~m}, 12 \mathrm{H}, \mathrm{H}-2{ }^{\prime}, \mathrm{H}-3^{\prime}, \mathrm{H}-4\right.$ ', $\left.\mathrm{Ar},=\mathrm{CH}\right) .{ }^{13} \mathrm{C} \mathrm{NMR}(75 \mathrm{MHz}$, DMSO- $\left.d_{6}\right) \delta(\mathrm{ppm})=42.45\left(\mathrm{~N}\left(\mathrm{CH}_{2}\right)_{4} \mathrm{~N}\right), 129.08\left(\mathrm{C}-2^{\prime}\right), 129.16(=\mathrm{CH}), 129.22(\mathrm{C}=), 129.55$ (C-3'), 129.82 (C-4'), 129.94 (C-1'); 130.41 (C=N), 159.56 (C=O). HRMS, m/z = 483.0918 found (calculated for $\mathrm{C}_{24} \mathrm{H}_{20} \mathrm{~N}_{4} \mathrm{O}_{2} \mathrm{NaS}_{2},[\mathrm{M}+\mathrm{Na}]^{+}$requires 483.0925). Anal. Calcd. for $\mathrm{C}_{24} \mathrm{H}_{20} \mathrm{~N}_{4} \mathrm{O}_{2} \mathrm{~S}_{2}$ : C, 62.59; $\mathrm{H}, 4.38$. Found: $\mathrm{C}, 62.56 ; \mathrm{H}, 4.41$.

(5Z,5'Z)-2,2'-(Piperazine-1,4-diyl)bis[5-(4-methoxybenzylidene)-1,3-thiazol-4(5H)-one] (1,4-Bis [(5Z)-5-(4-methoxybenzylidene)-4-oxo-4,5-dihydro-1,3-thiazol-2-yl]piperazine, 5b)

Prepared following General procedure III from 5-(4-methoxybenzylidene)-2-thioxothiazolidin-4-one (3b) (1.005 g, $4 \mathrm{mmol})$ and piperazine $4 \mathrm{a}(172 \mathrm{mg}, 2 \mathrm{mmol})$ at $80^{\circ} \mathrm{C}$ to give $\mathbf{5 b}$ in $36 \%$ yield as a yellow powder. $\mathrm{Mp}>260^{\circ} \mathrm{C} .{ }^{1} \mathrm{H}$ NMR $\left(300 \mathrm{MHz}, \mathrm{DMSO}-d_{6}\right) \delta$ $(\mathrm{ppm})=2.99\left(\mathrm{~s}, 8 \mathrm{H}, \mathrm{N}\left(\mathrm{CH}_{2}\right)_{4} \mathrm{~N}\right), 3.79\left(\mathrm{~s}, 6 \mathrm{H}, \mathrm{OCH}_{3}\right), 7.04(\mathrm{~d}, \mathrm{~J}=5 \mathrm{~Hz}, 4 \mathrm{H}, \mathrm{H}-3$ ', Ar), 7.16 $(\mathrm{s}, 2 \mathrm{H},=\mathrm{CH}), 7.43(\mathrm{~d}, \mathrm{~J}=8.7 \mathrm{~Hz}, 4 \mathrm{H}, \mathrm{H}-2 \mathrm{\prime}, \mathrm{Ar}) .{ }^{13} \mathrm{C} \mathrm{NMR}\left(75 \mathrm{MHz}, \mathrm{DMSO}-\mathrm{d}_{6}\right) \delta(\mathrm{ppm})=$

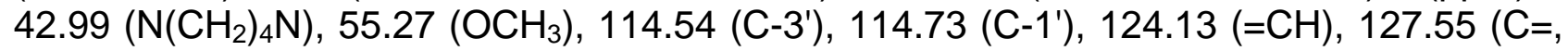
C-5), 131.29 (C-2'), 131.38 (C-4'), 132.14 (C=N, C-2), 159.56 (C=O, C-4). HRMS, m/z = 521.1317 found (calculated for $\mathrm{C}_{26} \mathrm{H}_{25} \mathrm{~N}_{4} \mathrm{O}_{4} \mathrm{~S}_{2},[\mathrm{M}+\mathrm{H}]^{+}$requires 521.1310). Anal. Calcd. for $\mathrm{C}_{26} \mathrm{H}_{24} \mathrm{~N}_{4} \mathrm{O}_{4} \mathrm{~S}_{2}$ : C, 59.98; $\mathrm{H}, 4.65$. Found: $\mathrm{C}, 59.87 ; \mathrm{H}, 4.64$.

\section{(5Z,5'Z)-2,2'-(Piperazine-1,4-diyl)bis[5-(1,3-benzodioxol-5-ylmethylene)-1,3-thiazol- 4(5H)-one]}

(1,4-Bis[(5Z)-5-(1,3-benzodioxol-5-ylmethylene)-4-oxo-4,5-dihydro-1,3-thiazol2-yl]piperazine, 5c)

Prepared following General procedure III from 5-(1,3-benzodioxol-5-ylmethylidene)-2thioxothiazolidin-4-one (3c) (1.061 g, $4 \mathrm{mmol})$ and piperazine $4 \mathrm{aa}(172 \mathrm{mg}, 2 \mathrm{mmol})$ in hexane $(2 \mathrm{ml})$ at $120^{\circ} \mathrm{C}$ to give $5 \mathrm{a}$ in $15 \%$ yield as a brown powder. $\mathrm{Mp}>260^{\circ} \mathrm{C}$. ${ }^{1} \mathrm{H} \mathrm{NMR}$ $\left(300 \mathrm{MHz}, \mathrm{DMSO}-d_{6}\right) \delta(\mathrm{ppm})=3.02\left(\mathrm{~s}, 8 \mathrm{H}, \mathrm{N}\left(\mathrm{CH}_{2}\right)_{4} \mathrm{~N}\right), 6.11\left(\mathrm{~s}, 4 \mathrm{H}, \mathrm{OCH}_{2} \mathrm{O}\right), 7.02-7.18$ $\left(\mathrm{m}, 6 \mathrm{H}, \mathrm{H}-2^{\prime}, \mathrm{H}-5^{\prime}, \mathrm{H}-6{ }^{\prime}, \mathrm{Ar}\right), 7.58(\mathrm{~s}, 2 \mathrm{H},=\mathrm{CH}) .{ }^{13} \mathrm{C} N M R\left(75 \mathrm{MHz}, \mathrm{DMSO}-d_{6}\right) \delta(\mathrm{ppm})=$ $52.72\left(\mathrm{~N}^{\left(\mathrm{CH}_{2}\right)}{ }_{4} \mathrm{~N}\right), 101.88\left(\mathrm{OCH}_{2} \mathrm{O}\right), 108.71\left(\mathrm{C}-2{ }^{\prime}\right), 125.13\left(\mathrm{C}^{\prime} 5^{\prime}\right), 126.04(\mathrm{C}=, \mathrm{C}-5), 127.86$ $\left(\mathrm{C}-1^{\prime}\right), 130.41\left(\mathrm{C}^{\prime} 6^{\prime}\right), 144.21(=\mathrm{CH}), 146.02(\mathrm{C}=\mathrm{N}, \mathrm{C}-2), 148.11\left(\mathrm{C}-4^{\prime}\right), 148.78\left(\mathrm{C}-3^{\prime}\right)$, $182.22(\mathrm{C}=\mathrm{O}, \mathrm{C}-4)$. HRMS, $\mathrm{m} / \mathrm{z}=571.0726$ found (calculated for $\mathrm{C}_{26} \mathrm{H}_{20} \mathrm{~N}_{4} \mathrm{O}_{6} \mathrm{NaS}_{2}$, $[\mathrm{M}+\mathrm{Na}]^{+}$requires 571.0722). Anal. Calcd. for $\mathrm{C}_{26} \mathrm{H}_{20} \mathrm{~N}_{4} \mathrm{O}_{6} \mathrm{~S}_{2}$ : C, 56.92; $\mathrm{H}, 3.67$. Found: C, 56.96; H, 3.71. 
(5Z,5'Z)-2,2'-[Piperazine-1,4-diylbis(propane-3,1-diylimino)]bis[5-(4-methoxybenzylidene)-1,3-thiazol-4(5H)-one] (1,4-Bis(3-\{[(5Z)-5-(4-methoxybenzylidene)-4-oxo-4,5-dihydro-1,3-thiazol2-yl]amino\}propyl)piperazine, 5d)

Prepared following General procedure III from 5-(4-methoxybenzylidene)-2-thioxothiazolidin-4-one (3b) (1.005 g, $4 \mathrm{mmol}$ ) and 1,4-bis(3-aminopropyl)piperazine (4b) (0.4 g, $2 \mathrm{mmol}$ ) in hexane $(2 \mathrm{ml})$ at $120^{\circ} \mathrm{C}$ to give $5 \mathrm{~d}$ in $25 \%$ yield as a yellow powder. $\mathrm{Mp}=220$ $222^{\circ} \mathrm{C}$. ${ }^{1} \mathrm{H}$ NMR $\left(300 \mathrm{MHz}, \mathrm{DMSO}_{-} d_{6}\right) \delta(\mathrm{ppm})=1.68$ (quint, $\mathrm{J}=6.9 \mathrm{~Hz}, 4 \mathrm{H}$, $\left.\mathrm{NCH}_{2} \mathrm{CH}_{2} \mathrm{CH}_{2} \mathrm{~N}\left(\mathrm{CH}_{2}\right)_{4} \mathrm{~N}\right), 2.37\left(\mathrm{t}, \mathrm{J}=6.7 \mathrm{~Hz} ; 4 \mathrm{H}, \mathrm{NCH}_{2} \mathrm{CH}_{2} \mathrm{CH}_{2} \mathrm{~N}\left(\mathrm{CH}_{2}\right)_{4} \mathrm{~N}\right), 2.57(\mathrm{~s}, 8 \mathrm{H}$, $\left.\mathrm{NCH}_{2} \mathrm{CH}_{2} \mathrm{CH}_{2} \mathrm{~N}\left(\mathrm{CH}_{2}\right)_{4} \mathrm{~N}\right), 2.84\left(\mathrm{t}, \mathrm{J}=7.1 \mathrm{~Hz}, 4 \mathrm{H} ; \mathrm{NCH}_{2} \mathrm{CH}_{2} \mathrm{CH}_{2} \mathrm{~N}\left(\mathrm{CH}_{2}\right)_{4} \mathrm{~N}\right), 3.79(\mathrm{~s}, 6 \mathrm{H}$, $\left.\mathrm{OCH}_{3}\right), 7.02\left(\mathrm{~d}, \mathrm{~J}=8.8 \mathrm{~Hz}, 4 \mathrm{H}, \mathrm{H}-3^{\prime}, \mathrm{Ar}\right), 7.18(\mathrm{~s}, 2 \mathrm{H}$; $=\mathrm{CH}), 7.44\left(\mathrm{~d}, \mathrm{~J}=8.8 \mathrm{~Hz}, 4 \mathrm{H}, \mathrm{H}-2^{\prime}\right.$, Ar). ${ }^{13} \mathrm{C}$ NMR $\left(75 \mathrm{MHz}\right.$, DMSO- $\left.d_{6}\right) \delta(\mathrm{ppm})=23.39\left(\mathrm{NCH}_{2} \mathrm{CH}_{2} \mathrm{CH}_{2} \mathrm{~N}\left(\mathrm{CH}_{2}\right){ }_{4} \mathrm{~N}\right), 37.41$ $\left(\mathrm{NCH}_{2} \mathrm{CH}_{2} \mathrm{CH}_{2} \mathrm{~N}\left(\mathrm{CH}_{2}\right)_{4} \mathrm{~N}\right), \quad 52.03 \quad\left(\mathrm{NCH}_{2} \mathrm{CH}_{2} \mathrm{CH}_{2} \mathrm{~N}(\underline{\mathrm{CH}})_{4} \mathrm{~N}\right), \quad 54.42$ $\left(\mathrm{NCH}_{2} \mathrm{CH}_{2} \mathrm{CH}_{2} \mathrm{~N}\left(\mathrm{CH}_{2}\right)_{4} \mathrm{~N}\right), 55.27\left(\mathrm{OCH}_{3}\right), 114.58\left(\mathrm{C}-3^{\prime}\right), 125.04(=\mathrm{CH}), 127.28\left(\mathrm{C}-1^{\prime}\right)$, 130.99 (C=), 131.44 (C-2'), 159.77 (C-4'), 201.61 (C=N, C-2), 206.74 (C=O, C-4). HRMS, $\mathrm{m} / \mathrm{z}=635.2396$ found (calculated for $\mathrm{C}_{32} \mathrm{H}_{39} \mathrm{~N}_{6} \mathrm{O}_{4} \mathrm{~S}_{2},[\mathrm{M}+\mathrm{H}]^{+}$requires 635.1819). Anal. Calcd. for $\mathrm{C}_{32} \mathrm{H}_{38} \mathrm{~N}_{6} \mathrm{O}_{4} \mathrm{~S}_{2}$ : C, 60.54; $\mathrm{H}, 6.03$. Found: $\mathrm{C}, 60.57 ; \mathrm{H}, 6.07$.

(5Z,5'Z)-2,2'-[Piperazine-1,4-diylbis(propane-3,1-diylimino)]bis[5-(1,3-benzodioxol5-ylmethylene)-1,3-thiazol-4(5H)-one]

(1,4-Bis(3-\{[(5Z)-5-(1,3-benzodioxol-5-ylmethylene)-4-oxo-4,5-dihydro-1,3-thiazol2-yl]amino\}propyl)piperazine, 5e)

Prepared following General procedure III from 5-(1,3-benzodioxol-5-ylmethylidene)-2thioxothiazolidin-4-one (3c) (1.061 g, $4 \mathrm{mmol}$ ) and 1,4-bis(3-aminopropyl)piperazine (4b) $(0.4 \mathrm{~g}, 2 \mathrm{mmol})$ in hexane $(2 \mathrm{ml})$ at $120^{\circ} \mathrm{C}$ to give $5 \mathrm{e}$ in $15 \%$ yield as a brown powder. $\mathrm{Mp}$ $=222-224^{\circ} \mathrm{C} .{ }^{1} \mathrm{H}$ NMR $\left(300 \mathrm{MHz}, \mathrm{DMSO}-d_{6}\right) \delta(\mathrm{ppm})=1,69$ (quint, $\mathrm{J}=6.9 \mathrm{~Hz}, 4 \mathrm{H}$, $\left.\mathrm{NCH}_{2} \mathrm{CH}_{2} \mathrm{CH}_{2} \mathrm{~N}\left(\mathrm{CH}_{2}\right)_{4} \mathrm{~N}\right), 2.37\left(\mathrm{t}, \mathrm{J}=6.6 \mathrm{~Hz}, 4 \mathrm{H}, \mathrm{NCH}_{2} \mathrm{CH}_{2} \mathrm{CH}_{2} \mathrm{~N}\left(\mathrm{CH}_{2}\right)_{4} \mathrm{~N}\right), 2.37(\mathrm{~s}, 8 \mathrm{H}$, $\left.\mathrm{NCH}_{2} \mathrm{CH}_{2} \mathrm{CH}_{2} \mathrm{~N}\left(\mathrm{CH}_{2}\right)_{4} \mathrm{~N}\right), 2.83\left(\mathrm{t}, \mathrm{J}=7.1 \mathrm{~Hz}, 4 \mathrm{H}, \mathrm{NCH}_{2} \mathrm{CH}_{2} \mathrm{CH}_{2} \mathrm{~N}\left(\mathrm{CH}_{2}\right)_{4} \mathrm{~N}\right), 6.07$ (s, 4H, $\left.\mathrm{OCH}_{2} \mathrm{O}\right), 7.02\left(\mathrm{~m}, 6 \mathrm{H}, \mathrm{H}-2^{\prime}, \mathrm{H}-5^{\prime}, \mathrm{H}-6{ }^{\prime}, \mathrm{Ar}\right), 7.14(\mathrm{~s}, 2 \mathrm{H},=\mathrm{CH}) .{ }^{13} \mathrm{C} N M R(75 \mathrm{MHz}$, DMSO$\left.d_{6}\right) \delta(\mathrm{ppm})=23.47\left(\mathrm{NCH}_{2} \underline{\mathrm{CH}_{2}} \mathrm{CH}_{2} \mathrm{~N}\left(\mathrm{CH}_{2}\right)_{4} \mathrm{~N}\right), 37.45\left(\mathrm{NCH}_{2} \mathrm{CH}_{2} \mathrm{CH}_{2} \mathrm{~N}\left(\mathrm{CH}_{2}\right)_{4} \mathrm{~N}\right), 52.22$ $\left(\mathrm{NCH}_{2} \mathrm{CH}_{2} \mathrm{CH}_{2} \mathrm{~N}\left(\mathrm{CH}_{2}\right)_{4} \mathrm{~N}\right), 54.54\left(\mathrm{NCH}_{2} \mathrm{CH}_{2} \mathrm{CH}_{2} \mathrm{~N}\left(\mathrm{CH}_{2}\right)_{4} \mathrm{~N}\right), 101.52\left(\mathrm{OCH}_{2} \mathrm{O}\right), 108.74(\mathrm{C}-$ 2'), $108.82\left(\mathrm{C}^{\prime} 5^{\prime}\right), 124.21$ (C-6'), $124.84(=\mathrm{CH}), 147.72\left(\mathrm{C}-4^{\prime}\right), 147.89$ (C-3'), 182.66 (C=, C-5), 202.14 (C=N, C-2), 206.72 ( $\mathrm{C}=\mathrm{O}, \mathrm{C}-4)$. HRMS, $\mathrm{m} / \mathrm{z}=663.1981$ found (calculated for $\mathrm{C}_{32} \mathrm{H}_{35} \mathrm{~N}_{6} \mathrm{O}_{6} \mathrm{~S}_{2},[\mathrm{M}+\mathrm{H}]^{+}$requires 663.7790). Anal. Calcd. for $\mathrm{C}_{32} \mathrm{H}_{34} \mathrm{~N}_{6} \mathrm{O}_{6} \mathrm{~S}_{2}$ : C, 57.99; $\mathrm{H}$, 5.17. Found: $\mathrm{C}, 57.92 ; \mathrm{H}, 5.16$.

\section{In vitro kinase inhibition assays}

Buffer A: $10 \mathrm{mM} \mathrm{MgCl}$, $1 \mathrm{mM}$ EGTA, $1 \mathrm{mM}$ DTT, $25 \mathrm{mM}$ Tris-HCl pH 7.5, $50 \mu \mathrm{g}$ heparin/ml. Buffer C: $60 \mathrm{mM}$ ß-glycerophosphate, $15 \mathrm{mM}$ p-nitrophenylphosphate, $25 \mathrm{mM}$ Mops (pH 7.2), $5 \mathrm{mM}$ EGTA, $15 \mathrm{mM} \mathrm{MgCl}_{2}, 1 \mathrm{mM}$ DTT, $1 \mathrm{mM}$ sodium vanadate, $1 \mathrm{mM}$ phenylphosphate. Kinase activities were assayed in Buffer $\mathrm{A}$ or $\mathrm{C}$, at $30^{\circ} \mathrm{C}$, at a final ATP concentration of $15 \mu \mathrm{M}$. Blank values were subtracted and activities expressed in \% of the maximal activity, i.e. in the absence of inhibitors. Controls were performed with appropriate dilutions of DMSO. The kinase peptide substrates were obtained from Millegen (Labege, France). DYRK1A and DYRK2 (human, recombinant, expressed in E. coli as a GST fusion protein) were purified by affinity chromatography on glutathione-agarose and assayed in buffer A (+ $0.5 \mathrm{mg} \mathrm{BSA} \mathrm{/ml)} \mathrm{using} \mathrm{Woodtide} \mathrm{(KKISGRLSPIMTEQ)} \mathrm{(1.5} \mathrm{\mu g/assay)} \mathrm{as} \mathrm{a}$ 
substrate, in the presence of $15 \mu \mathrm{M}\left[\mathrm{\gamma}^{-33} \mathrm{P}\right]$ ATP $(3,000 \mathrm{Ci} / \mathrm{mmol} ; 10 \mathrm{mCi} / \mathrm{ml})$ in a final volume of $30 \mu \mathrm{l}$. After $30 \mathrm{~min}$ incubation at $30^{\circ} \mathrm{C}$, the reaction was stopped by harvesting onto P81 phosphocellulose papers (Whatman) using a FilterMate harvester (Packard) and were washed in $1 \%$ phosphoric acid. Scintillation fluid was added and the radioactivity was measured in a Packard counter. CLK1 and CLK3 (human, recombinant, expressed in E. coli as GST fusion proteins) were assayed in buffer A (+ $0.15 \mathrm{mg} \mathrm{BSA} / \mathrm{ml})$ with RS peptide (GRSRSRSRSRSR) (1 $\mu \mathrm{g} /$ assay). CDK5/p25 (human, recombinant) was prepared as previously described [26]. Its kinase activity was assayed in buffer $\mathrm{C}$, with $1 \mu \mathrm{g}$ histone $\mathrm{H} 1$ $/ \mathrm{ml}$. GSK-3 $\alpha / \beta$ (porcine brain, native) was assayed in Buffer A and using a GSK-3 specific substrate (GS-1: YRRAAVPPSPSLSRHSSPHQSpEDEEE) ( $p S$ stands for phosphorylated serine) [20].

\section{Cell culture and survival assays}

Skin diploid fibroblastic cells were provided by BIOPREDIC International Company (Rennes, France). Caco-2 cells and Huh7 cells were obtained from the ECAC collection. Cells were grown according to ECAC recommendations. The RLEC-F1 clone was derived from an established rat biliary epithelial cell line as previously described [21]. The toxicity test of the compounds on these cells was as follows: $4 \times 10^{3}$ cells were seeded in 96 multiwell plates and left for $24 \mathrm{~h}$ for attachment, spreading, and growing. Then they were exposed for $48 \mathrm{~h}$ to increasing concentrations of the compounds, ranging from 0.1 to $25 \mu \mathrm{L}$ in a final volume of $80 \mu \mathrm{L}$ of culture medium. They were then fixed with $4 \%$ paraformaldehyde solution and the nuclei were stained with Hoechst 3342 and counted using automated imaging analysis (Simple PCl software).

\section{Acknowledgement}

One of us (K. W. C.) wishes to thank the "Agence Universitaire de la Francophonie AUF, contract $\mathrm{N}^{\circ} 0486$ " for a research fellowship. Financial support of this program carried out under the French National Cancer Institute "Cancéropôle Grand Ouest" by contracts PRIR 04-8390 and ACI 04-2254, is gratefully acknowledged.

\section{Authors' Statement}

\section{Competing Interests}

The authors declare no conflict of interest.

\section{References}

[1] Mentgen T, Steuer C, Klein CD.

Privileged Scaffolds or Promiscuous Binders: A comparative Study on Rhodanines and Related Heterocycles in Medicinal Chemistry.

J Med Chem. 2012; 55: 743-753.

http://dx.doi.org/10.1021/jm201243p

[2] Nasr M.N.A., Said S-A.

Novel 3,3a,4,5,6,7-Hexahydroindazole and Arylthiazolylpyrazoline derivatives as Anti-inflammatory Agents.

Arch Pharm. 2003; 336: 551-559.

http://dx.doi.org/10.1002/ardp.200300796 
[3] Hu Y, Helm J-S, Chen L, Ginsberg C, Gross B, Kraybill B, Tiyanont K, Fang X, Wu T, Walker S. Identification of selective inhibitors for the glycosyltransferase MurG via high-throughput screening. Chem Biol. 2004; 11: 703-711. http://dx.doi.org/10.1016/j.chembiol.2004.02.024

[4] Martin L, Rabasseda X, Castaner J.

Darbufelone Mesilate.

Drugs Future. 1999; 24: 853-857.

http://dx.doi.org/10.1358/dof.1999.024.08.534526

[5] Johnson A-R, Marletta M-A, Dyer R-D.

Slow-binding inhibition of human prostaglandin endoperoxide synthase-2 with darbufelone, an isoform-selective antiinflammatory di-tert-butyl phenol.

Biochemistry 2001; 40: 7736-7745.

http://dx.doi.org/10.1021/bi002343f

[6] Inagaki M, Tsuri T, Jyoyama H, Ono T, Yamada K, Kobayashi M, Hori Y, Arumura A, Yasui K, Ohno K, Kakudo S, Koizumi K, Suzuki R, Kato M, Kawai S, Matsumoto S.

Novel antiarthritic agents with 1,2-isothiazolidine-1,1-dioxide ( $\gamma$-sultam) skeleton: cytokine suppressive dual inhibitors of cyclooxygenase-2 and 5-lipoxygenase.

J Med Chem. 2000; 43: 2040-2048.

http://dx.doi.org/10.1021/jm9906015

[7] Teraishi F, Wu S, Zhang L, Guo W, Davis J-J, Dong F, Fang B.

Identification of a novel synthetic thiazolidin compound capable of inducing c-Jun $\mathrm{NH} 2$-terminal kinase-dependent apoptosis in human colon cancer cells.

Cancer Res. 2005; 65: 6380-6387.

http://dx.doi.org/10.1158/0008-5472.CAN-05-0575

[8] Cutshall N-S, O'Day C, Prezhdo M.

Rhodanine derivatives as inhibitors of JSP-1.

Bioorg Med Chem Lett. 2005; 15: 3374-3382

http://dx.doi.org/10.1016/j.bmcl.2005.05.034

[9] Hotta N, Akanuma Y, Kawamori R, Matsuoka K, Oka Y, Shichiri M, Toyata T, Nakoshima M, Yoshimura I, Sakamoto N, Shigeta Y.

Long-Term clinical effects of Epalrestat, an aldose reductase inhibitor, on diabetic peripheral neuropathy: The 3-year, multicenter, comparative aldose reductase inhibitor-diabetes complications trial.

Diabetes Care. 2006; 29: 1538-1541.

http://dx.doi.org/10.2337/dc05-2370

[10] Zhang J, Yang P-L, Gray N-S.

Targeting cancer with small molecule kinase inhibitors.

Nat Rev Cancer. 2009; 9: 28-39.

http://dx.doi.org/10.1038/nrc2559

[11] Galons H, Oumata N, Meijer L.

Cyclin-dependent kinase inhibitors: a survey of recent patent literature.

Exp Opin Ther Patents. 2010, 20: 377-404.

http://dx.doi.org/10.1517/13543770903524284

[12] Druker B-J, Talpaz M, Resta D-J, Peng B, Buchdunger E, Ford J-M, Lydon, N-B, Kantarjian H, Capdeville R, Ohnojones S, C.L. Sawyers C-L.

Efficacy and Safety of a Specific Inhibitor of the BCR-ABL Tyrosine Kinase in Chronic Myeloid Leukemia.

N Engl J Med. 2001; 344: 1031-1037.

http://dx.doi.org/10.1056/NEJM200104053441401

[13] Bazureau J-P, Draye M; eds.

Ultrasound and Microwave: Recent advances in organic chemistry.

Transworld Research Signpost, Kerala, 2011.

ISBN: 978-81-7895-532-2 
[14] Guihéneuf S, Paquin L, Carreaux F, Durieu E, Meijer L, Bazureau J-P. An efficient approach to dispacamide $A$ and its derivatives.

Org Biomol Chem. 2012; 10: 978-987. http://dx.doi.org/10.1039/c1ob06161e

[15] Bourahla K, Derdour A, Rahmouni M, Carreaux F, Bazureau J-P. A practical access to novel 2-amino-5-arylidene-1,3-thiazol-4(5H)-ones via sulfur/nitrogen displacement under solvent-free microwave irradiation. Tetrahedron Lett. 2007, 48: 5785-5789. http://dx.doi.org/10.1016/j.tetlet.2007.06.078

[16] Kimura R, Kamino, K, Yamamoto M, Nuripa A, Kida T, Kazui H, Hashimoto R, Tanaka T, Kudo T, Yamagata H, Tabara Y, Miki T, Akatsu H, Kosaka K, Funakoshi E, Nishitomi K, Sakaguchi G, Kato A, Hattori $\mathrm{H}$, Uema $\mathrm{T}$, Takeda $\mathrm{M}$.

The DYRK1A gene, encoded in chromosome 21 Down syndrome critical region, bridges between $\beta$ amyloid production and tau phosphorylation in Alzheimer disease.

Hum Mol Genet. 2007; 16: 15-23.

http://dx.doi.org/10.1093/hmg/ddl437

[17] Debdab M, Carreaux F, Renault S, Soundararajan M, Federov O, Lozach O, Babault L, Baratte B, Ogawa Y, Hagiwara M, Einsenreich A, Rauch U, Knapp S, Meijer L, Bazureau J-P.

Design, synthesis and biological evaluation of leucettines, a class of potent CLK and DYRK kinases inhibitors derived from the marine sponge leucettamine B. Modulation of alternative RNA splicing. J Med Chem. 2011; 54: 4172-4186. http://dx.doi.org/10.1021/jm200274d

[18] Besyadetskaya El, Kuz'min Y.

Synthesis and properties of bicyclic compounds containing piperazine and thiazolidine rings Farmatsevt Zhurnal (Kiev). 1973; 28: 34-36.

[19] Besyadetskaya El.

Synthesis of 5-substituted N,N-bis(2-thiazolidin-4-one-2-yl)piperazines Farmatsevt Zhurnal (Kiev). 1972; 27: 30-32.

[20] Primot A, Baratte B, Gompel M, Borgne A, Liabeuf S, Romette J-L, Costantini F, Meijer L. Purification of GSK-3 by affinity chromatography on immobilised axin.

Protein Expression Purif. 2000; 20: 394-404. http://dx.doi.org/10.1006/prep.2000.1321

[21] Nakabayashi H, Taketssa K, Miyano K, Yamane T, Sato J.

Growth of Human Hepatoma Cell Lines with Differentiated Functions in Chemically Defined Medium Cancer Res. 1982; 42: 3858-3863.

http://www.ncbi.nlm.nih.gov/pubmed/6286115 